\title{
Energy Dissipation in Coronal Loops: Statistical Analysis of Intermittent Structures in Magnetohydrodynamic Turbulence
}

\author{
Laura F. Morales ${ }^{1,2}$, Pablo Dmitruk ${ }^{1,3}$, and Daniel O. Gómez ${ }^{1,4}$ (1) \\ ${ }^{1}$ Departamento de Física, Facultad de Ciencias Exactas y Naturales, UBA, Argentina; Imorales@df.uba.ar \\ 2 INFIP, UBA CONICET, Buenos Aires, Argentina \\ ${ }^{3}$ IFIBA, UBA CONICET, Buenos Aires, Argentina \\ ${ }^{4}$ IAFE, UBA CONICET, Buenos Aires, Argentina \\ Received 2019 December 30; revised 2020 March 25; accepted 2020 March 27; published 2020 May 8
}

\begin{abstract}
The power-law energy distribution observed in dissipation events ranging from flares down to nanoflares has been associated either to intermittent turbulence or to self-organized criticality. Despite the many studies conducted in recent years, it is unclear whether these two paradigms are mutually exclusive or they are complementary manifestations of the complexity of the system. We numerically integrate the magnetohydrodynamic equations to simulate the dynamics of coronal loops driven at their bases by footpoint motions. After a few photospheric turnover times, a stationary turbulent regime is reached, displaying a broadband power spectrum and a dissipation rate consistent with the cooling rates of the plasma confined in these loops. Our main goal is to determine whether the intermittent features observed in this turbulent flow can also be regarded as manifestations of self-organized criticality. A statistical analysis of the energy, area, and lifetime of the dissipative structures observed in these simulations displays robust scaling laws. We calculated the critical exponents characterizing the avalanche dynamics, and the spreading exponents that quantify the growth of these structures over time. In this work we also calculate the remaining critical exponents for several activity thresholds and verify that they satisfy the conservation relations predicted for self-organized critical systems. These results can therefore be regarded as a bona fide test supporting that the stationary turbulent regimes characterizing coronal loops also correspond to states of self-organized criticality.
\end{abstract}

Unified Astronomy Thesaurus concepts: Interplanetary turbulence (830); Solar coronal heating (1989)

\section{Introduction}

In recent decades it has been thoroughly demonstrated that energy dissipation in turbulent magnetic plasma is strongly intermittent both in space and time (Zelenyi et al. 2015) and (Matthaeus et al. 2015). This peculiarity has already been observed in diverse natural and simulated environments such as astrophysical plasmas like the solar corona, the solar wind, and Earth's magnetosphere (for a complete review see Aschwanden 2013), fusion plasmas (Plunk \& Tatsuno 2011), and numerical simulations (Dmitruk \& Gómez 1997; Dmitruk et al. 1998).

Flares are explosive events occurring in the solar corona in which energies as high as $10^{32} \mathrm{erg}$ are released in tens of minutes. The flaring region is associated with the emission of radiation across the entire electromagnetic spectrum (soft $\mathrm{X}$-rays and hard X-rays) and is also intimately associated with the acceleration of particles and coronal mass ejections (Webb \& Howard 2012). Since the first observations in the late 1980s and early 1990s, solar flares have shown some striking features such as power-law spectra for the total energy released, peak energy, and the duration of flares (see, for instance, Crosby et al. 1993; Aschwanden \& Parnell 2002; Podladchikova \& Lefebvre 2006, and Fletcher et al. 2011 for a review of recent observations). These results were generally explained using Parker's model (Parker 1983 and Parker 1988) in the form of two different paradigms: plasma turbulence (Dmitruk \& Gómez 1997) or coronal self-organized criticality (Lu \& Hamilton 1991 and Morales \& Charbonneau 2008a). These are not the only mechanisms proposed to heat the coronal plasma. For instance, Alfvén waves have also been invoked to serve this purpose (Klimchuk 2006). Observationally, the question of which accounts for most of the dissipation still remains elusive (Yang et al. 2018).

Additionally, more than a decade ago Uritsky and collaborators (Uritsky et al. 2007), using Solar and Heliospheric Observatory data of extreme ultraviolet images of the solar corona and applying typical statistical methods of selforganized criticality (SOC) and intermittent turbulence, showed that the abovementioned data simultaneously exhibited features of both regimes: power-law avalanche statistics as well as multiscaling of structure functions for spatial activity. Those observations led them to conclude that both SOC and turbulent intermittency are the manifestations of a single complex dynamical process that ultimately explains the flaring phenomena.

Turbulent numerical models of the solar corona producing intermittent energy release have been used to identify avalanche features that could be compared both to observational data and to results from cellular automata models (Dmitruk \& Gómez 1997 and Buchlin et al. 2003). This evidence leads to the following question: is it possible to identify representative SOC traits in turbulent simulations of flare modeling?

Over the last 25 years, the SOC community has produced a number of tools to perform bona fide tests of presumed SOC data in order to be able to separate real SOC states from mere power law-like behavior (Muñoz et al. 1999; Morales \& Charbonneau 2008b). In this work we used a previously developed turbulent numerical simulation of solar flares (Dmitruk \& Gómez 1997) to calculate the usual spreading exponents in order to test the SOC hypothesis to be able to 
bring new responses to the question of the coexistence of criticality and turbulence in the solar corona.

Section 2 presents the equations assumed to describe flaring phenomena in the solar corona, the numerical code that we use, and the results obtained, as well as the mechanism for detecting dissipative structures. Section 3 characterizes the structures and the presentation of the SOC tools used to test their behavior, as well as the results from these tests. In Section 4 we discuss the limits of SOC tests we perform and list our conclusions.

\section{Numerical Simulations and Detection of Dissipative Structures}

\subsection{MHD Simulations}

The internal dynamics of a coronal loop with a uniform magnetic field $\boldsymbol{B}=B_{0} \hat{z}$, length $L$, and transverse section $(2 \pi l) \times(2 \pi l)$ can be modeled by reduced magnetohydrodynamic (RMHD) equations (Strauss 1976)

$$
\begin{gathered}
\partial_{t} a=v_{\mathrm{A}} \partial_{z} \psi+[\psi, a]+\eta \nabla_{\perp}^{2} a \\
\partial_{t} w=v_{\mathrm{A}} \partial_{z} j+[\psi, w]-[a, j]+\nu \nabla_{\perp}^{2} w
\end{gathered}
$$

where $v_{\mathrm{A}}=B_{0} / \sqrt{4 \pi \rho}$ is the Alfvén speed, $\nu$ is the kinematic viscosity, $\eta$ is the plasma resistivity, $\psi$ is the stream function, and $a$ is the vector potential. The fluid vorticity is $w=-\nabla_{\perp}^{2} \psi$, $j=-\nabla_{\perp}^{2} a$ is the electric current density, and $[u, v]=z \cdot \nabla_{\perp} u \times \nabla_{\perp} v$ is the regular Poisson bracket. For given horizontal photospheric motions imposed at the end plates of the loop (i.e., plates $z=0$ and $z=L$ ), transverse velocity and magnetic field components develop in the interior of the loop, given by $\boldsymbol{u}=\nabla_{\perp} \times(\hat{z} \psi)$ and $\boldsymbol{b}=\nabla_{\perp} \times(\hat{z} a)$. The RMHD equations can be interpreted as describing a set of twodimensional MHD systems stacked along the loop axis and interacting among themselves through the $v_{\mathrm{A}} \partial_{z}$ terms (see, for instance, Hendrix \& van Hoven 1996). For simplicity, hereafter we study the evolution of a generic two-dimensional slice of a loop. We therefore model the $v_{\mathrm{A}} \partial_{z}$ terms in Equations (1)-(2) as external forces. To this end, we assume the vector potential to be independent of $z$ and the stream function to interpolate linearly between $\psi(z=0)=0$ and $\psi(z=L)=\Psi$, where $\Psi$ $(x, y, t)$ is the stream function for the photospheric velocity field. As a result, these assumptions imply $v_{\mathrm{A}} \partial_{z} \psi=v_{\mathrm{A}} \Psi / L$ (in Equation (1)) and $v_{\mathrm{A}} \partial_{z} j=0$ (in Equation (2)). These expressions correspond to an idealized scenario where the magnetic stress distributes uniformly throughout the loop, and the resulting $2 \mathrm{D}$ equations are

$$
\begin{gathered}
\partial_{t} a=[\psi, a]+\eta \nabla_{\perp}^{2} a+\frac{v_{\mathrm{A}}}{L} \Psi \\
\partial_{t} w=[\psi, w]-[a, j]+\nu \nabla_{\perp}^{2} w
\end{gathered}
$$

where the last term in Equation (3) represents the external forcing of the photospheric motions. This type of forcing promotes the generation of vortex flows to imitate granule motions. Its amplitude corresponds to a velocity field intensity $u_{\mathrm{ph}}$, a typical granule size $l_{\mathrm{ph}}$ and a correlation time $1 / w$. For these simulations, we chose $1 / w$ to be a long timescale (in fact much longer than any other timescale in the system) so that the photospheric time remains much longer than the Alfvén transit time for loops. There is also another timescale involved in the forcing, related with the amplitude and length scale of the vortex motions, namely $t_{\mathrm{ph}}=l_{\mathrm{ph}} / u_{\mathrm{ph}}$, representing the period of photospheric granules. This timescale $t_{\mathrm{ph}}$ enters directly into the dynamics of the system via the amplitude of the forcing in Equation (3) (see more details in Dmitruk \& Gómez 1997).

More specifically, we choose a forcing term, which is a narrow band in wavenumber space, to imitate the granular subphotospheric motions

$$
\Psi_{k}= \begin{cases}\Psi_{0} \cos (\omega t) & \text { if } 3<k<4 \\ 0 & \text { elsewhere. }\end{cases}
$$

Even though similar approaches have been followed in the literature (for instance, by Einaudi et al. 1996 and Georgoulis et al. 1998), we chose a narrowband and nonrandom forcing to make sure that the broadband energy spectra and the signatures of intermittency that we obtained are exclusively determined by the nonlinear nature of the MHD equations (Dmitruk \& Gómez 1997).

For the numerical integration, we convert Equations (3)-(4) into a dimensionless version, choosing $l$ and $L$ as the units for transverse and longitudinal distances. Note that the large disparity $(l \ll L)$ between the transverse and longitudinal length scales is crucial for the RMHD description (see, for instance, Strauss 1976). We choose $u_{p h}=\sqrt{\Psi_{0}}$ as the unit for velocities. The dimensionless dissipation coefficients are $\nu_{0}=\nu /\left(l u_{\mathrm{ph}}\right)$ and $\eta_{0}=\eta /\left(l u_{\mathrm{ph}}\right)$. The numerical simulations were performed on a square box, assuming periodic boundary conditions. The code is of the pseudospectral type, with twothirds de-aliasing (Canuto et al. 1988). Spatial derivatives are computed in Fourier space with the aid of a fast Fourier transform algorithm, and the nonlinear products are computed in physical space. The temporal integration scheme is a secondorder Runge-Kutta. The pseudospectral method allows us to achieve an almost exact energy balance over our extended time simulations.

We performed $512 \times 512$ numerical simulations for extended periods of time for three different intensities of the external forcing $\left(\Psi_{0}=0.1,0.2,0.3\right)$, which in turn correspond to different photospheric velocities. In Figure 1 we display the dissipation rate versus time for the case $\Psi_{0}=0.2$ and for 1000 photospheric turnover times. This figure shows a fast relaxation (after several turnover times) to a stationary turbulent regime, characterized by intermittent bursts of dissipation. Figure 2 shows the energy power spectra for the various intensities of forcing, as labeled. A $k^{-5 / 3}$ law (see Kolmogorov 1991) is also displayed for reference. Even though our simulations have moderate spatial resolution, all of them are consistent with the slope predicted by Kolmogorov for homogeneous, isotropic, and stationary turbulence.

\subsection{Detection of Dissipative Structures}

Our simulations produced localized dissipative structures of different sizes and durations that can be interpreted as nanoflare events. In order to define a coherent spatiotemporal structure we have adopted the following criteria.

We calculate the electric current density $j(x, y, t)$, which clearly displays (see the left panel of Figure 3 ) the spatial and temporal intermittencies of a turbulent regime. For each simulation, we search for regions in the $(x, y, t)$ domain where $|j(x, y, t)|$ exceeds a preset threshold $j_{c}$, i.e., where the energy dissipation rate surpasses $\eta j_{c}^{2}$. In order to single out each 


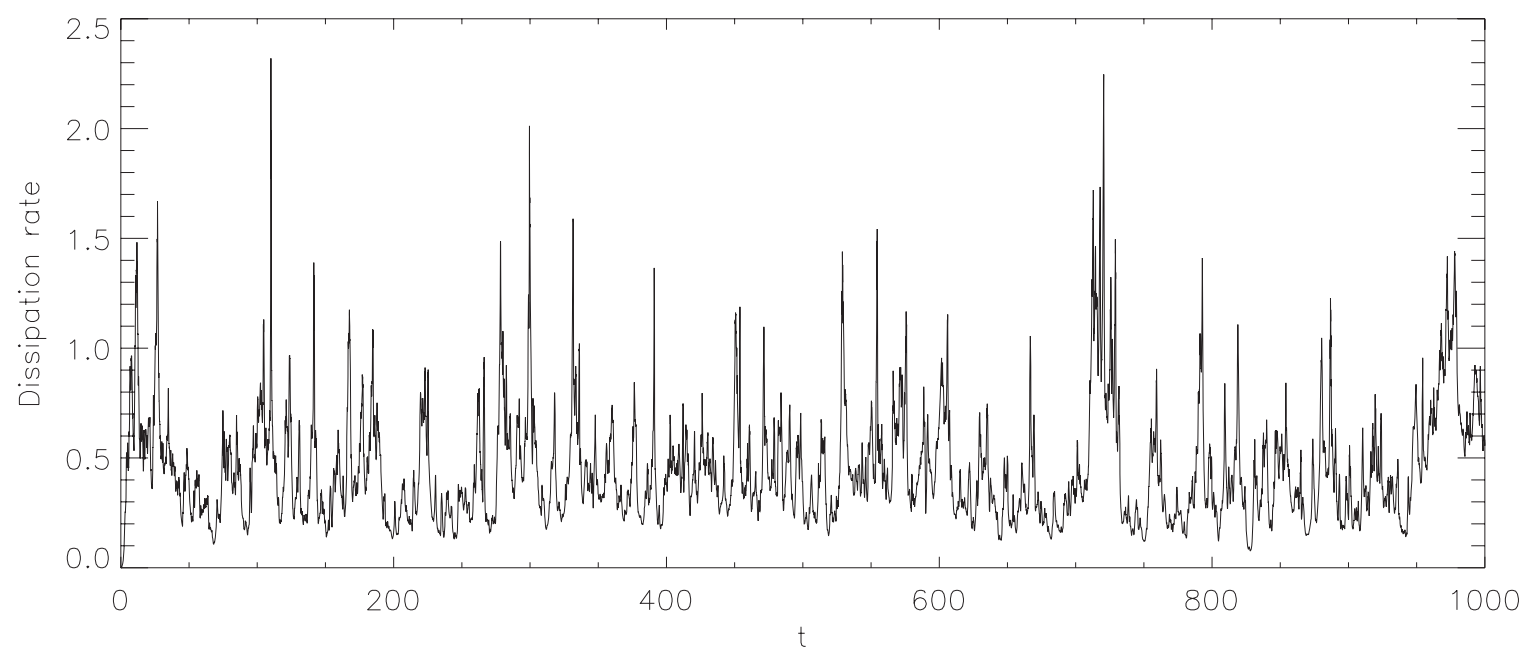

Figure 1. Dissipation rate vs. time for run $f=0.3$.

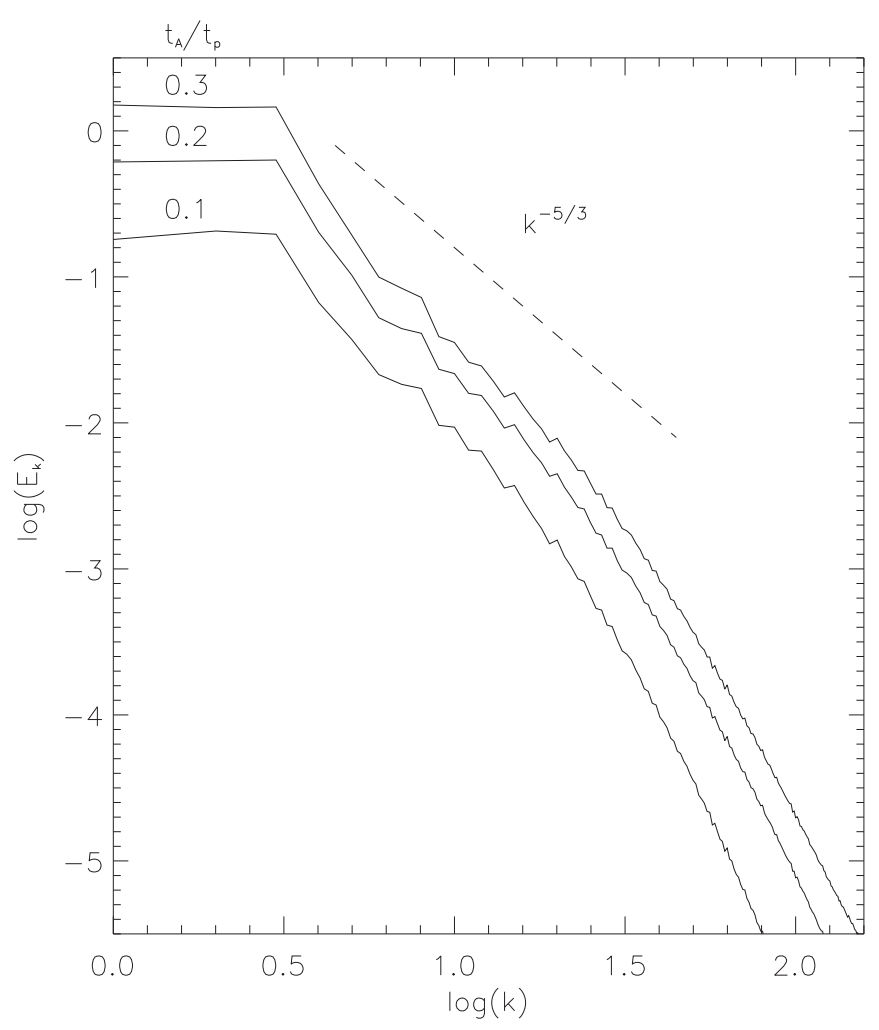

Figure 2. Energy spectra for three different simulations given by $f=0.1$; $0.2 ; 0.3$.

dissipative structure (i.e., each nanoflare) we used the HoshenKopelman algorithm (Hoshen \& Kopelman 1976), which is a numerical technique that finds and identifies clusters of homogeneous patches in a matrix. Cluster membership is defined by first neighboring adjacency. With this routine we transformed the energy dissipation rate, which is continuously distributed in space and time, into a discrete and numerable set of dissipative structures or nanoflares in the $(x, y, t)$ domain.

Those clusters can in turn be interpreted as avalanche-like events of liberation of energy similar to the ones produced by cellular automata models (Lu \& Hamilton 1991; Charbonneau et al. 2001; Morales \& Charbonneau 2008a; Hendrix \& van Hoven 1996). Figure 3 (right) also shows the particular dissipative structure identified within the white rectangle in the left frame at four different times $(t=829.5,829.8,830.1$, 830.4 ) once the thresholding technique has been applied. In this particular case the structure reduces and migrates along the zoom region. Eventually the structure fades and the avalanche stops.

The choice of the precise value of the threshold current $j_{c}$ is crucial. If $j_{c}$ is too low (for instance, of the order of the square root of the mean value of $j^{2}$ ) it will not be possible to discriminate structures and if $j_{c}$ is too high (close to the maximum of $j$ ), we might only obtain very few and short-lived structures missing a significant amount of the dissipated energy. Statistically, we want to end up having a robust sample of nanoflares events. The value of $j_{c}$ was chosen in such a way that over $60 \%$ of the time average dissipated energy is confined in somewhat less than $8 \%$ of the box.

\section{Characterizing the Dissipative Structures}

We have assumed that a current sheet structure that arises at time $t_{0}$ and disappears by time $t_{f}$ is like the avalanche produced by a cellular automaton. Thus, these structures can be characterized using one (or both) of the two methods used for avalanches in self-organized models: avalanche exponents (for a thorough summary see Charbonneau et al. 2001) and spreading exponents (for a theoretical introduction see Muñoz et al. 1999 and for astrophysical applications see Uritsky et al. 2001 and Morales \& Charbonneau 2009).

\subsection{Statistics of Structures}

The first test we perform in order to assess the SOC behavior of the system is to study the statistics of the ensemble of structures. We have built the probability distribution functions (PDFs) for three of the physical quantities that characterize dissipative events: the lifetime of the structure $T=t_{f}-t_{0}$; the total dissipated energy $E$; and the peak dissipation rate $P$. In Figure 4 we present the observed distributions. As expected, they take the form of power laws: $\operatorname{PDF}(z) \propto z^{-\alpha_{z}}$ (in this case $z$ being $E, P$, or $T$ ) spanning six to seven orders of magnitude and the values of $\alpha_{z}$ are not only in very good agreement with solar flare observations (see for example, Benz 2017), but also compare very well with most of the values obtained by direct numerical simulations (Dmitruk \& Gómez 1997) and the exponents obtained by various cellular automata experiments 

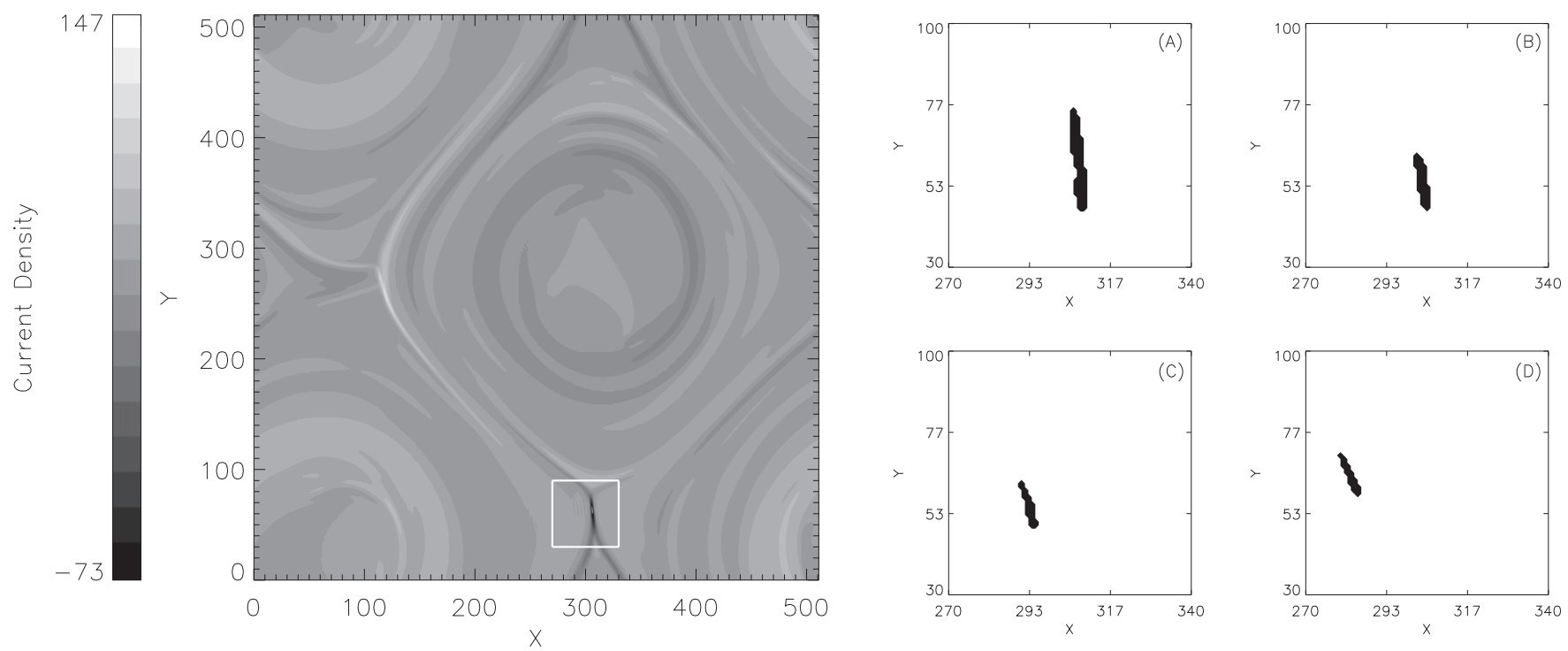

Figure 3. Left: contours of current density at an arbitrary time $(t=829.5)$ in the stationary regime of the simulation for $\Psi_{0}=0.3$. Black and white represent minimum and maximum values, while gray means close to zero. Right: zoom of the region enclosed by the white rectangle (see left) displaying the dissipative structure determined by the thresholding technique at times $t=829.5,829.8,830.1,830.4$.
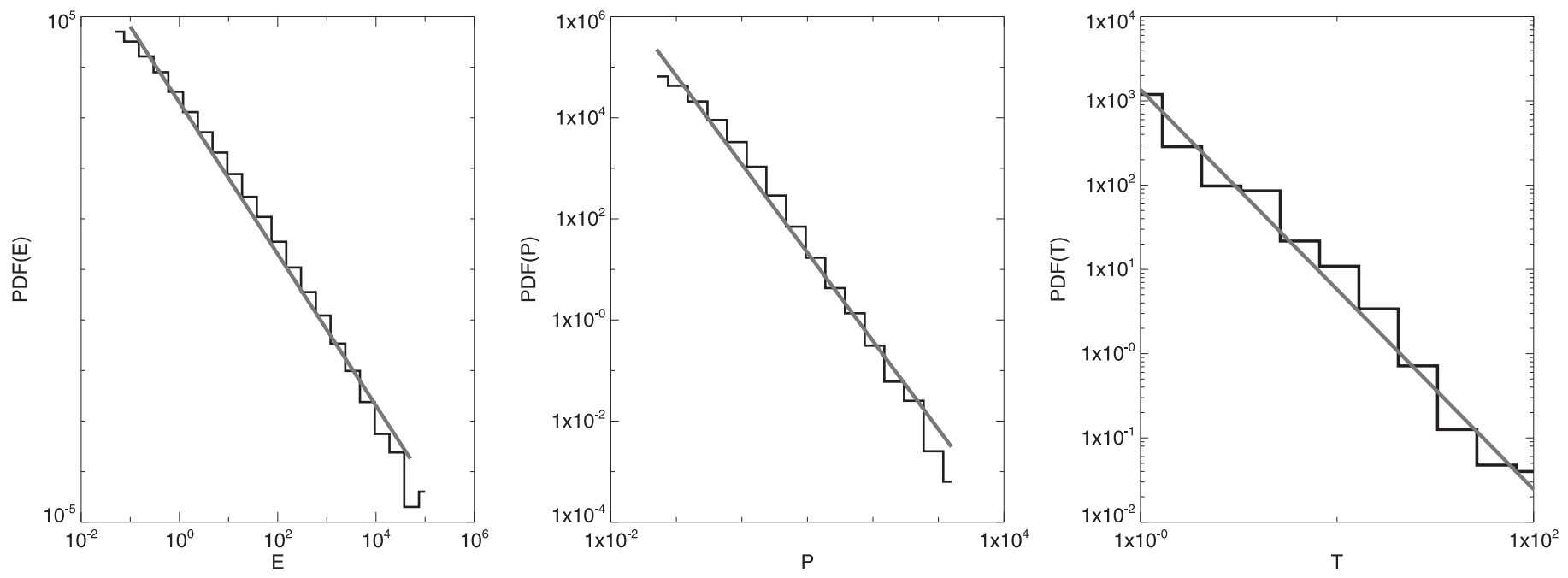

Figure 4. Probability distribution functions of structure-size measures $E, P$, and $T$ in a representative $x 6$ iterations run of model $f=0.30$.

(Morales \& Charbonneau 2008a; Aschwanden 2013). We have listed in Table 1 the power-law indices obtained for different values of the preset forcing. The power-law forms of their PDFs, and the constancy of the associated power-law exponents as the driving force intensity increases, point to a lack of a typical scale for avalanches, a sine qua non feature of SOC systems.

\subsection{Spreading Exponents}

In the study of systems with absorbing states (a typical case is percolation) it is usual to measure, at the critical point, the spreading of an initial seed of activity throughout an otherwise absorbing configuration: these are known as the spreading exponents (Muñoz et al. 1999). They provide the most accurate and unambiguous determination of the critical point in such systems (Grassberger \& de la Torre 1979).

In the last 15 years, the apparent similarities between avalanching and absorbing systems have been acknowledged and spreading exponents analysis is increasingly used to
Table 1

Simulation Parameters and Global Results

\begin{tabular}{lccc}
\hline \hline Forcing & $\alpha_{E}$ & $\alpha_{P}$ & $\alpha_{T}$ \\
\hline 0.10 & $1.55 \pm 0.12$ & $1.74 \pm 0.16$ & $2.30 \pm 0.15$ \\
0.20 & $1.53 \pm 0.12$ & $1.72 \pm 0.17$ & $2.38 \pm 0.14$ \\
0.30 & $1.57 \pm 0.12$ & $1.78 \pm 0.16$ & $2.36 \pm 0.12$ \\
\hline
\end{tabular}

determine the critical point in the system. For applications to space phenomena, see, for instance, Morales \& Charbonneau (2008c) for the case of solar flares, and Uritsky et al. (2001) for magnetic substorms. The spreading of avalanches (dissipative structures) in a self-organized system can be characterized in terms of the number of active sites $N(t)$ (in a statistical sense) at a certain time $t$ and the probability $P(t)$ that an avalanche is still active at time $t$. In the vicinity of the critical state both quantities scale as follows:

$$
N(t)=t^{\eta} \quad P(t)=t^{-\delta} .
$$


Table 2

Simulation Parameters and Spreading Exponent Results

\begin{tabular}{lcccc}
\hline \hline Forcing & $\eta$ & $\beta$ & $\delta$ & $\kappa$ \\
\hline 0.10 & 0.28 & 1.73 & 1.53 & 2.75 \\
0.20 & 0.26 & 1.76 & 1.59 & 2.64 \\
0.30 & 0.24 & 1.71 & 1.57 & 2.68 \\
\hline
\end{tabular}

From these definitions we can derive that the total number of active sites having a lifetime $T$ scales as

$$
n_{s}=T^{\eta+\delta}
$$

As a consequence, the size of an avalanche that dies after a time $T$ can be obtained by integrating expression (7), leading to

$$
S=T^{1+\eta+\delta} \text {. }
$$

If we define $\kappa=1+\eta+\delta$ we get an exponent that can be calculated independently of $\eta$ and $\delta$, giving rise to a firstconsistency test for the spreading exponents.

The other spreading exponent that can be defined in terms of $\eta$ and $\delta$ is $\beta$, which can be derived from the expression of the avalanche size probability distribution $P(s)$. Since the size $(S)$ and the duration $T$ of avalanches are not correlated, the probability that an avalanche will reach a size $S$ before ending is

$$
P(s)=\int_{t_{\min }}^{t_{\max }} P(s \mid t) D(t) \mathrm{d} t
$$

where $t_{\min }$ and $t_{\max }$ are the upper and lower duration bounds of size $s$ avalanches, $D(t) \sim t^{-\delta-1}$ is the probability that an avalanche (structure) will die between $t$ and $t+d t$, and $P(s \mid t)$ is the conditional probability of an avalanche having reached size $s$ at time $t$ since onset. Since $P(s \mid t)$ is bell-shaped and reaches its maximum value at $t \sim 1 / s^{1+\eta+\delta}$ it can be shown that $P(s)$ scales as (for a complete derivation see Muñoz et al.

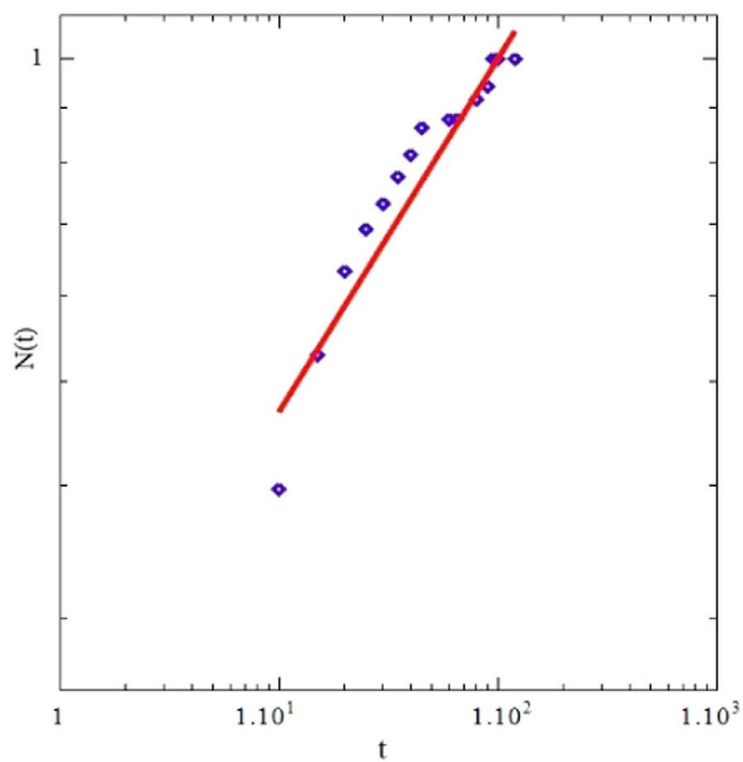

1999)

$$
P(s) \propto s^{-\beta}, \quad \beta=\frac{1+\eta+2 \delta}{1+\eta+\delta},
$$

where $\beta=\frac{1+\eta+2 \delta}{1+\eta+\delta}$ is the second spreading exponent that can be calculated independently and also in terms of $\eta$ and $\beta$ allowing us to test the found critical state of the system for consistency. With the data gathered in this work we are able to compute $N(t)$ and $P(t)$ functions for the three different simulations performed (see Table 2). In Figure 5 we present an illustrative plot obtained for a specific driving force intensity. The calculated values for $\delta$ yield results that are very similar to those reported in the realization of the OrzagTang vortex generalized to MHD (Uritsky et al. 2010) and the findings for an anisotropic SOC model for solar flares (Morales \& Charbonneau 2008b). The estimation of $\eta$ is more complicated, therefore the results obtained carry somewhat larger errors. Nevertheless, our estimations remain close to those produced by Morales \& Charbonneau (2008a).

\section{Concluding Remarks}

Whether or not MHD turbulence exhibits avalanching behavior is a longstanding question (see, for instance, Charbonneau et al. 2001) and several studies have explored this issue using solar flare data (Uritsky et al. 2007 and Abramenko et al. 2008) or numerical simulations (Zhdankin et al. 2015).

In this work we calculated both the avalanche exponents and the spreading exponents that characterize intermittent dissipative structures in an MHD incompressible turbulent simulation.

We found that the statistical properties of the defined structures associated with the electric current density-total dissipated energy $E$, peak dissipation rate $P$, and lifetime $T-$ are all in good agreement with indices obtained in the case of solar flare observations (Aschwanden \& Parnell 2002) and also with values obtained for cellular automata models of solar flares (Morales \& Charbonneau 2008a). These results suggest that the intermittent structures that emerge from the numerical

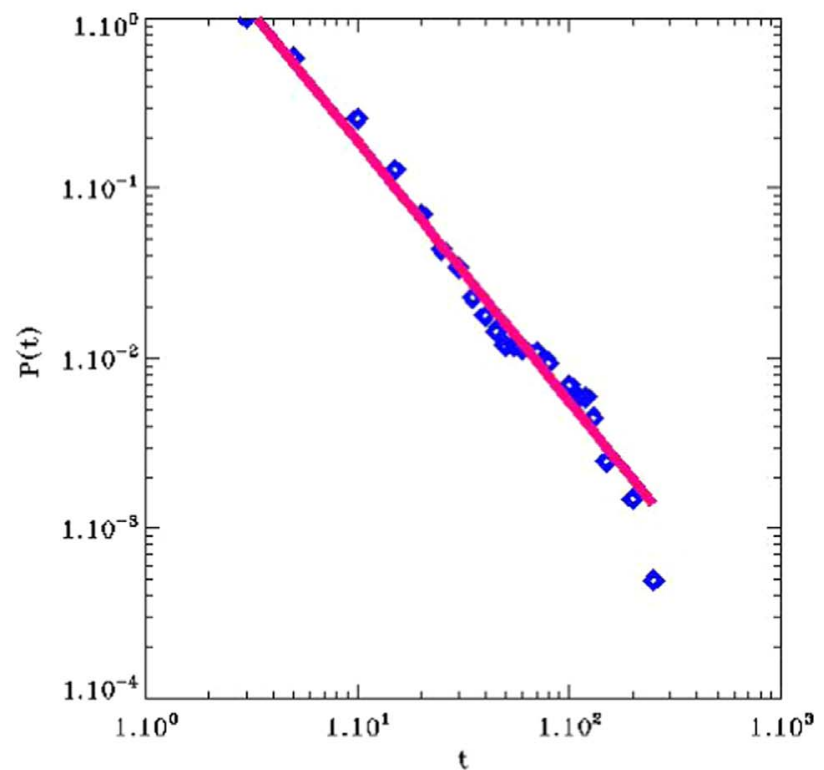

Figure 5. Correlation plots of the number of active sites vs. time and the surviving probability for a run with driving $f=0.30$. 
simulations can indeed be treated as the computational representation of flaring activity in the solar corona.

With this in mind, we calculated typical spreading exponents $\eta$ and $\delta$ and verified that they satisfy the mutual numerical relationship expected from SOC systems.

These findings support the conjecture that the solar corona can be described by a model that simultaneously exhibits robust signatures of SOC and intermittent turbulence.

Accordingly, following our statistical analysis, we infer that the nanoflares responsible for the heating of coronal active regions can be interpreted both as dissipative structures of MHD turbulence as well as SOC avalanches of reconnecting magnetic loops.

The existence of intermittent turbulence and SOC features has not only been observed in magnetized fluids like that in the corona (Uritsky et al. 2007, Abramenko et al. 2008 and Karakatsanis et al. 2013), but also in geophysical fluids (Smyth et al. 2019), suggesting that the findings reported in this paper might be a confirmation of a more comprehensive result.

Research reported in this publication was supported by Agencia Nacional de Promoción Científica y Tecnológica grant: PICT-1707-2015 and Consejo Nacional de Investigaciones Científicas y Ténicas-PIP 11220150100324CO.

\section{ORCID iDs}

Daniel O. Gómez (iD https://orcid.org/0000-0002-4935-4358

\section{References}

Abramenko, V. I., Valentyna, Y., \& Wang, H. 2008, ApJ, 681, 1669 Aschwanden, M. J. 2013, Self-Organized Criticality Systems (Berlin: Open Academic Press)

Aschwanden, M. J., \& Parnell, C. E. 2002, ApJ, 572, 1048

Benz, A. 2017, LRSP, 14, 12

Buchlin, E., Aletti, V., Galtier, S., et al. 2003, A\&A, 406, 1061
Canuto, C., Yousuff Hussaini, M., Quarteroni, A., \& Zang, T. A. 1988, Spectral Methods in Fluid Dynamics (Berlin: Springer),

Charbonneau, P., McIntosh, S. W., Liu, H.-L., \& Bogdan, T. J. 2001, SoPh, 203, 321

Crosby, N., Aschwanden, M., \& Dennis, B. 1993, AdSpR, 13, 179

Dmitruk, P., \& Gómez, D. O. 1997, ApJL, 484, L83

Dmitruk, P., Gómez, D. O., \& DeLuca, Edward E. 1998, ApJ, 505, 974

Einaudi, G., Velli, M., Politano, H., \& Pouquet, A. 1996, ApJL, 457, L113

Fletcher, L., Dennis, B. R., Hudson, H. S., et al. 2011, SSRv, 159, 19

Georgoulis, M. K., Velli, M., \& Einaudi, G. 1998, ApJ, 497, 957

Grassberger, P., \& de la Torre, A. 1979, AnPhy, 122, 373

Hendrix, D. L., \& van Hoven, G. 1996, ApJ, 467, 887

Hoshen, J., \& Kopelman, R. 1976, PhRvB, 14, 3438

Karakatsanis, L. P., Pavlos, G. P., \& Xenakis, M. N. 2013, PhyA, 392, 3920

Klimchuk, J. A. 2006, SoPh, 234, 41

Kolmogorov, A. N. 1991, RSPSA, 434, 9

Lu, E. T., \& Hamilton, R. J. 1991, ApJL, 380, L89

Matthaeus, W. H., Wan, M., Servidio, S., et al. 2015, RSPTA, 373, 20140154

Morales, L., \& Charbonneau, P. 2008a, ApJ, 682, 654

Morales, L., \& Charbonneau, P. 2009, ApJ, 698, 1893

Morales, L. F., \& Charbonneau, P. 2008b, GeoRL, 35, L04108

Muñoz, M. A., Dickman, R., Vespignani, A., \& Zapperi, S. 1999, PhRvE, 59,6175

Parker, E. N. 1983, ApJ, 264, 642

Parker, E. N. 1988, ApJ, 330, 474

Plunk, G. G., \& Tatsuno, T. 2011, PhRvL, 106, 165003

Podladchikova, O., \& Lefebvre, B. 2006, in IAU Symp. 233, Solar Activity and its Magnetic Origin, ed. V. Bothmer \& A. A. Hady (Cambridge: Cambridge Univ. Press), 481

Smyth, W., Nash, J., \& Moum, J. 2019, NatSR, 9, 3747

Strauss, H. R. 1976, PhFl, 19, 1411

Uritsky, V. M., Klimas, A. J., \& Vassiliadis, D. 2001, GeoRL, 28, 3809

Uritsky, V. M., Paczuski, M., Davila, J. M., \& Jones, S. I. 2007, PhRvL, 99, 025001

Uritsky, V. M., Pouquet, A., Rosenberg, D., Mininni, P. D., \& Donovan, E. F. 2010, PhRvE, 82, 056326

Webb, D. F., \& Howard, T. A. 2012, LRSP, 9, 3

Yang, K. E., Longcope, D. W., Ding, M. D., \& Guo, Y. 2018, NatCo, 9, 692

Zelenyi, L. M., Bykov, A. M., Uvarov, Y. A., \& Artemyev, A. V. 2015, JPIPh, 81,395810401

Zhdankin, V., Uzdensky, D. A., \& Boldyrev, S. 2015, PhRvL, 114, 065002 